\title{
Design of Broadband Dispersion Compensating Photonic Crystal Fiber
}

\author{
Md. Selim Habib, Md. Samiul Habib, S.M.A. Razzak, M. A. G. Khan \\ Department of Electrical \& Electronic Engineering, Rajshahi University of \\ Engineering \& Technology, Rajshahi-6204, Bangladesh. \\ E-mail: selim041073@yahoo.com, samiul.engieee@gmail.com, \\ razzak91@yahoo.com,agmagk@gmail.com
}

\begin{abstract}
This paper presents a triangular-lattice photonic crystal fiber for broadband dispersion compensation. The finite element method with perfectly matched absorbing layers boundary condition is used to investigate the guiding properties. The designed dispersion compensating fiber shows that it is possible to obtain a larger negative dispersion coefficient of $-360 \mathrm{ps} /(\mathrm{nm} . \mathrm{km})$ at $1.55 \mu \mathrm{m}$, better dispersion slope compensation, better compensation ratio in the entire telecommunication (1460-1640 $\mathrm{nm}$ ) band by using a modest number of design parameters and very simple cladding design.
\end{abstract}

Keywords: Photonic crystal fiber, chromatic dispersion, finite element method.

\section{Introduction}

Photonic crystal fibers (PCFs) [1] consisting of a central defect region surrounded by air holes running parallel to the fiber length have been one of the most interesting development in recent fiber optics. PCFs are usually made from pure silica, and so the guided modes are inherently leaky because the core index is the same as the index of the outer cladding region without air holes. Control of chromatic dispersion in PCFs is very important problem for realistic applications of optical fiber communications [2], dispersion compensation [3], and so on. Dispersion causes pulses to spread and has to be compensated in the long distance optical data transmission systems. One way to realize this is to use dispersion 
compensating fibers (DCFs), which are designed to have large negative dispersion. The negative dispersion coefficient of conventional fiber is about $\mathrm{D}=-100$ $-130 \mathrm{ps} /(\mathrm{nm} . \mathrm{km})$ at $1.55 \mu \mathrm{m}$ wavelength with high losses [4]. In order to minimize losses and reduce costs, the DCFs should be as short as possible and thus the magnitude of negative dispersion should be as large as possible [5-6]. In order to efficiently compensate the dispersion of all the frequencies of the DWDM, the negative dispersion of DCFs should span around wide spectrum and dispersion slope should be compensated at the same time.

Various designs of dispersion compensating PCFs (DC-PCFs) have been reported in [6-10] to date. Dual-concentric-core PCFs without Ge doping has been proposed [5-6]. It should be noted that PCFs is very useful for chromatic dispersion management and it is possible to obtain large negative dispersion coefficient without Ge doping. However, the available wavelength bandwidth for dispersion compensation of single mode fibers (SMFs) is very narrow because of the concave dispersion profile of dual-concentric-core fibers. Therefore it is necessary to compensate for the dispersion slope of SMFs as well for broadband operation. On the other hand some have been able to achieve small negative dispersion values, but bandwidth is limited [8]. Recently Erbium doped PCFs have been presented [9], which have fine dispersion-compensating characteristic, but the fabrication will be very difficult. With carefully chosen structural parameters dual-concentric-core fibers have been proposed for broadband dispersion compensation of SMFs [10], the problem due to Ge doping still remains.

In this paper, we present pure silica DC-PCFs for optimizing the dispersion compensation of standard SMFs in the entire telecommunication band (1460$1640 \mathrm{~nm}$ ) by using full-vector finite element method (FEM). From the numerical simulation results, it is found that it is possible to obtain larger negative dispersion, better dispersion slope, and better dispersion compensation ratio in the entire $\mathrm{S}+\mathrm{C}+\mathrm{L}$ band. Another main advantage is that, compared with previously presented DC-PCFs, the design procedure for this proposed DC-PCFs structure could be more efficient and easier because relatively fewer geometrical parameters are need to be optimized.

\section{Geometries of the Proposed DC-PCFs}

Fig. 1. depicts the transverse cross-section of DC-PCFs which contains six airhole rings. The material of the studied PCF is taken to be silica. The cladding is formed by a triangular-lattice of air holes. The air-hole pitch is labeled $\Lambda$ (the distance between the centers of neighbouring air holes). It has a pitch $\Lambda$, two types of air hole diameters $d_{1}$ and $d$. In the proposed structure, the diameter of the first air-hole ring $d_{l}\left(d>d_{1}\right)$ to obtain large negative dispersion. The diameter of 
other air hole rings $d$ is selected large for keeping low confinement loss level in the targeted region. The total number of air-hole rings is chosen to be six in order to simplify as much as possible the structural composition of the PCF which is shown in Fig. 1. In particular, the geometric parameters which characterize these triangular PCFs have been chosen to optimize the fiber length and the dispersion compensation over a wide wavelength range.

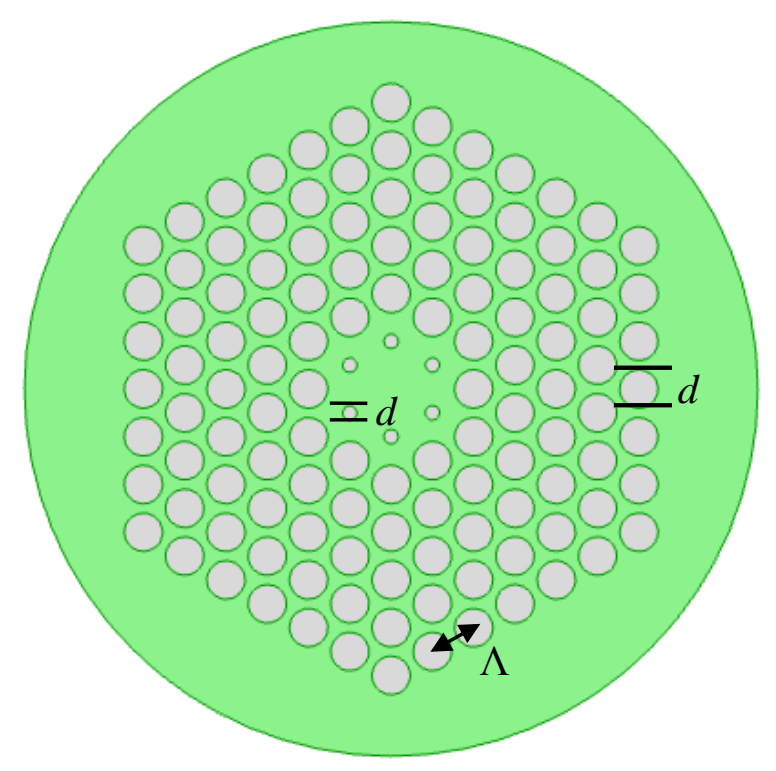

Fig. 1. Transverse cross-section of the six-ring DC-PCFs with pitch and air hole ring diameter.

\section{Numerical method}

The finite element method with perfectly matched boundary layers (PML) is used to investigate the guiding properties of PCFs. Using the FEM, the PCF crosssection, with the finite number of air holes is divided into homogeneous subspaces where Maxwell's equations are solved by accounting for the adjacent subspaces. These subspaces are triangles that allow a good approximation of the circular structures. Using the PML, from Maxwell's curl equations the following vectorial equation is obtained [12]

$$
\nabla \times\left([\mathrm{s}]^{-1} \nabla \times \mathrm{E}\right)-k_{0}^{2} n_{e f f}^{2}[\mathrm{~s}] \mathrm{E}=0
$$


where $\mathrm{E}$ is the electric field vector, $k_{0}$ is the wave number in the vacuum, $n_{\text {eff }}$ is the refractive index of the domain, $[\mathrm{s}]$ is the PML matrix, $[\mathrm{s}]^{-1}$ is the inverse matrix of [s]. Once the modal effective indices $n_{\text {eff }}$ is obtained by solving an eigen value problem using FEM, the chromatic dispersion $\mathrm{D}(\lambda)$ can be calculated given by [2].

\subsection{Chromatic dispersion}

The chromatic dispersion $D$ of PCFs is easily calculated from the $n_{\text {eff }}$ values vs. wavelength using the following [2]

$$
D(\lambda)=-\frac{\lambda}{c} \frac{d^{2} \operatorname{Re}\left[n_{e f f}\right]}{d \lambda^{2}}
$$

in $\mathrm{ps} /(\mathrm{nm} . \mathrm{km})$, where $\operatorname{Re}\left[n_{\text {eff }}\right]$ is the real part of effective refractive index $n_{\text {eff }}, \lambda$ is the wavelength, $\mathrm{c}$ is the velocity of light in vacuum. The material dispersion can be obtained from the three-term Sellmeier formula and it is directly included in the calculation. In PCFs, the chromatic dispersion $D$ is related to the additional design parameters like geometry of the air holes, pitch, and hole diameters. By optimizing these parameters, suitable guiding properties can be obtained.

\subsection{Dispersion Compensation}

Dispersion compensating fiber is a fiber that has the opposite dispersion of the fiber being used in the transmission system. It is used to nullify the dispersion caused by that fiber. The terms of broadband dispersion compensation [4]

$$
D_{S M F} \cdot L_{S M F}+D_{D C F} \cdot L_{D C F}=D_{T}
$$

where $D_{S M F}, D_{D C F}, L_{S M F}$, and $L_{D C F}$ are, respectively, the dispersion coefficients and the lengths of the single-mode and the dispersion compensating fibers. If the total compensation of the dispersion is required, the length of the DCFs $L_{D C F}$ is chosen so that total residual dispersion $D_{T}=0$. However, due to nonlinear effects and possible chirp in transmitter, full compensation is not always the optimum [11].

\subsection{Condition for Slope Compensation}

For multichannel high-speed WDM systems, dispersion compensation over a broad wavelength range is necessary. This means that besides the dispersion, it is 
also necessary to compensate for the dispersion slope. The total dispersion slope is

$$
S_{\text {slope }}=S_{S M F} \cdot L_{S M F}+S_{D C F} \cdot L_{D C F}
$$

where $S_{S M F}, S_{D C F}$ are the dispersion slopes of the SMFs and the DCFs respectively. As seen from Eq. (4), a negative dispersion slope of the DCFs is necessary in order to achieve slope compensation $\left(\mathrm{S}_{\text {slope }}=0\right)$. If the length of the DCFs is chosen to give full compensation $\left(D_{\text {res }}=0\right)$, then the condition for full slope compensation is that the relative dispersion slope (RDS) of the DCFs shall be equal to the relative dispersion slope of the standard SMFs

$$
R D S_{D C F}=R D S_{S M F}
$$

The relative dispersion slope is defined as the ratio of dispersion slope to dispersion

$$
R D S=S / D
$$

Again from Eq. (5) and Eq. (6), we can write

$$
S_{S M F} / D_{S M F}=S_{D C F} / D_{D C F}
$$

It is noticed that RDS value of standard SMFs is about $0.0036 \mathrm{~nm}^{-1}$ at $1.55 \mu \mathrm{m}$.

\subsection{Dispersion compensation ratio}

DCR $(\lambda)$ is the fraction of the SMF dispersion which the DCF compensates at a wavelength $\lambda$, that is

$$
\operatorname{DCR}(\lambda)=\left|\mathrm{D}_{\mathrm{SMF}}(\lambda) \cdot \mathrm{L}_{\mathrm{SMF}} / \mathrm{D}_{\mathrm{DCF}}(\lambda) \cdot \mathrm{L}_{\mathrm{DCF}}\right|
$$

\section{Dispersion Analysis of Broadband DC-PCFs}

\subsection{Large negative dispersion properties}

The guiding properties of PCFs with a triangular lattice of air-holes have been investigated as a function of the geometric characteristics that is the hole-to-hole spacing $\Lambda$ and the diameter $d_{l}$ and $d$ of the air-hole in the fiber cross-section.

Fig. 2 shows the plot of effective refractive index versus wavelength for different structural parameters. It is found that the effective refractive index decreases with the increase in wavelength and increases with increase of pitch, $\Lambda$, which is in good agreement with [14] 


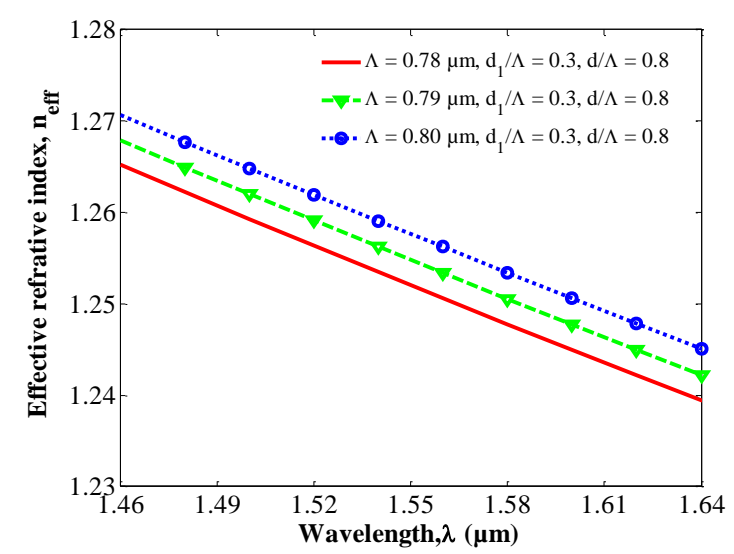

Fig. 2. Effective refractive index as a function of wavelength. The variation of effective refractive index with pitch $\Lambda$ and air-hole diameter $d_{l}$ and $d$ is also shown in the figure.

Fig. 3 shows the wavelength dependence properties of chromatic dispersion for the six-ring DC-PCFs in Fig. 1.

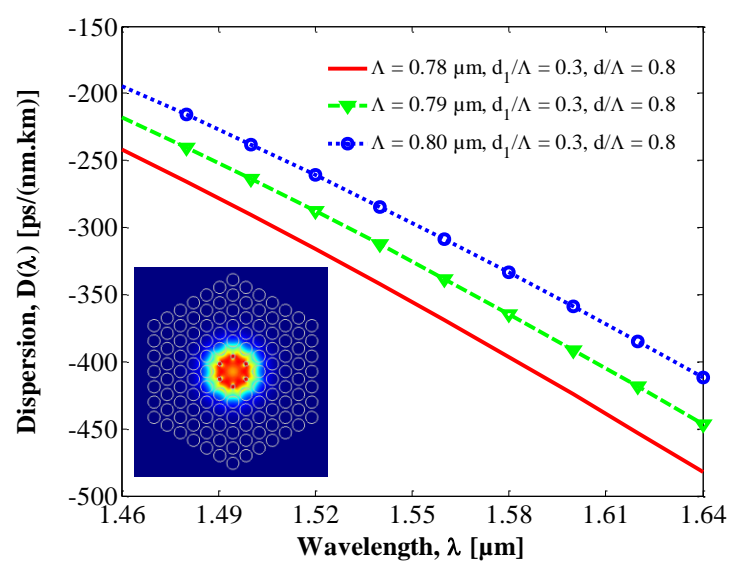

Fig. 3. Chromatic dispersion curves as a function of wavelength of the DC-PCFs with $d_{1} / \Lambda=0.3, d / \Lambda=0.8$ and different $\Lambda$ values. Insert shows the fundamental mode field at $1.55 \mu \mathrm{m}$.

Here we have considered three different types of parameter value to get large negative dispersion. In all cases, it is found that the proposed DC-PCFs shows large negative dispersion properties in the wavelength range 1.46-1.64 $\mu \mathrm{m}$. From the above results, it can be seen that the absolute values of the negative dispersion coefficient decrease appreciably as pitch increases. Therefore, in the practice, we can design broadband DC-PCFs with large negative dispersion coefficient by selecting appropriate parameters. All the dispersion curves presented in this paper is calculated for the fundamental mode of DC-PCFs. 


\subsection{Compensation ratio, residual dispersion, dispersion slope, and relative dispersion slope}

Fig. 4 represents the wavelength dependence properties of chromatic dispersion for the six-ring DC-PCFs in Fig. 1. Fig. 4 shows optimized chromatic dispersion of DC-PCFs for $d_{1} / \Lambda=0.3, d / \Lambda=0.8$ and $\Lambda=0.78 \mu \mathrm{m}$. It is found that the proposed DC-PCFs shows larger negative dispersion coefficients of around -240 to $-482 \mathrm{ps} /(\mathrm{nm} . \mathrm{km})$ in the wavelength range $1.46-1.64 \mu \mathrm{m}(180 \mathrm{~nm}$ band $)$.

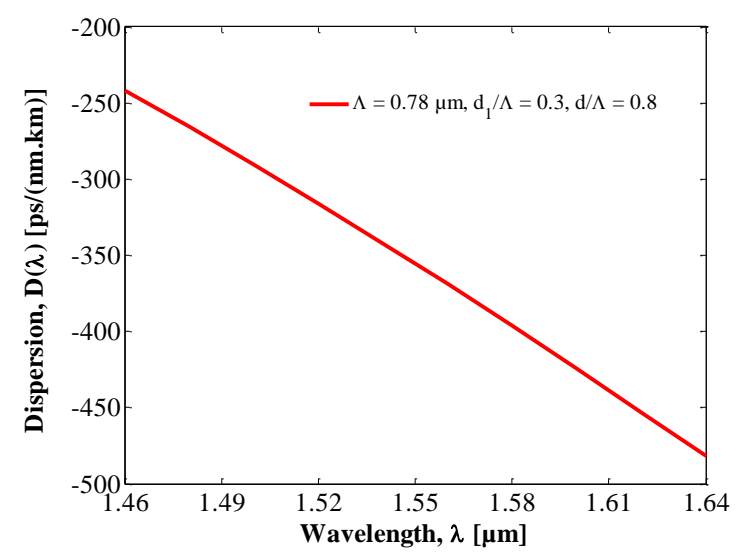

Fig. 4. Wavelength dependence of chromatic dispersion for the optimum design parameters: $d_{1} / \Lambda=0.3, d / \Lambda=0.8$ and $\Lambda=0.78 \mu \mathrm{m}$.

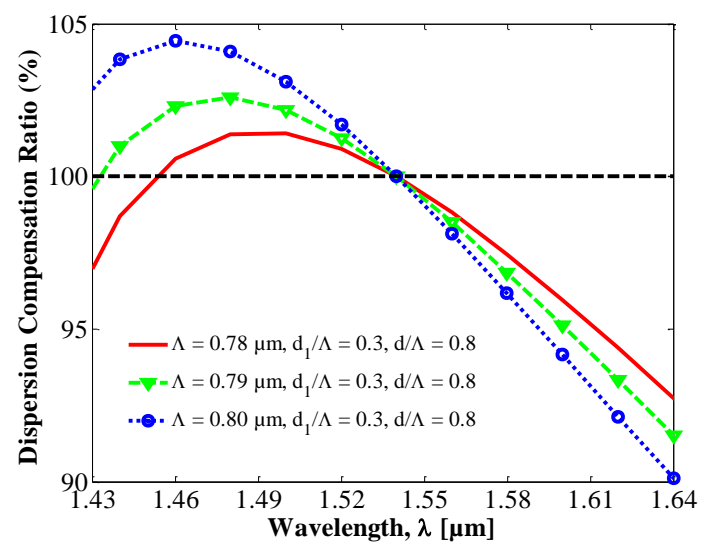

Fig. 5. The dispersion compensation ratio (DCR) versus wavelength. Here, the optimum design parameter is $d_{1} / \Lambda=0.3, d / \Lambda=0.8$ and $\Lambda=0.78 \mu \mathrm{m}$.

$\operatorname{DCR}(\lambda)$ evaluated for several triangulat PCFs is shown on $180 \mathrm{~nm}$ wavelength range for $d_{1} / \Lambda=0.3, d / \Lambda=0.8$ and different $\Lambda$ values in Fig. 5. The best 
compensation is obtained with the PCF with $d_{l} / \Lambda=0.3, d / \Lambda=0.8$ and $\Lambda=0.78$ $\mu \mathrm{m}$.

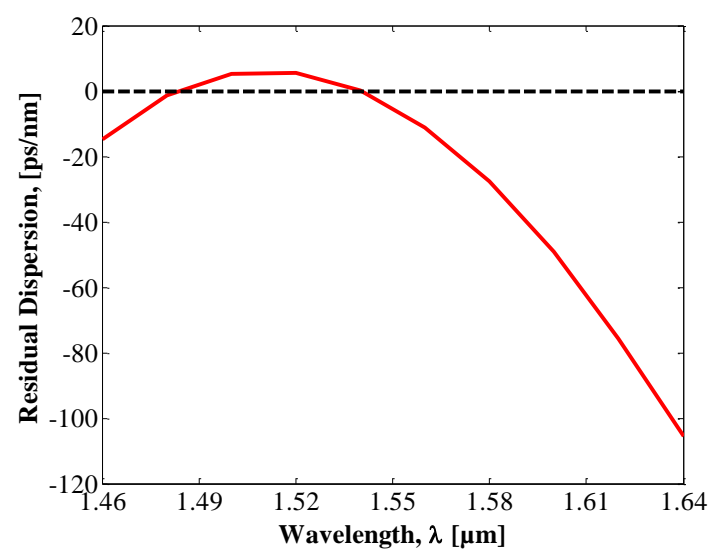

Fig. 6. Residual dispersion curve for the optimum design parameters: $d_{1} / \Lambda=0.3$, $d / \Lambda=0.8$ and $\Lambda=0.78 \mu \mathrm{m}$. Here corresponding residual dispersion curve after compensating $80 \mathrm{~km}$ SMF link by $3.9 \mathrm{~km}$ long optimized PCF.

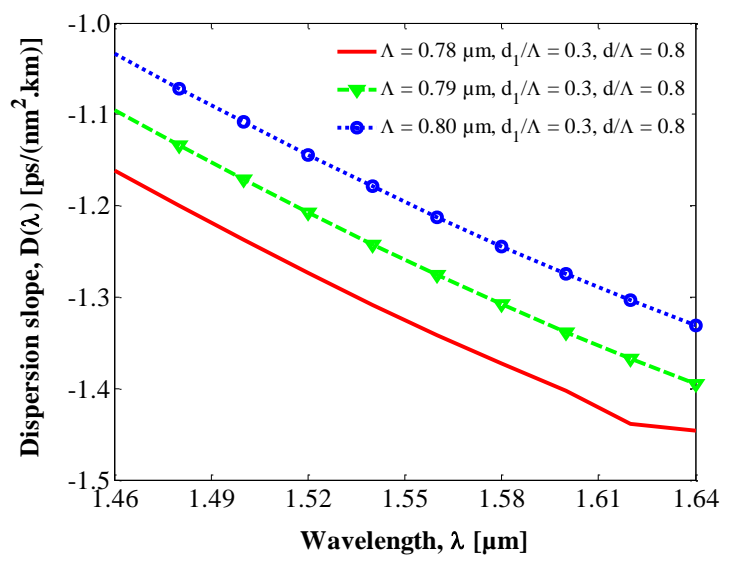

Fig. 7. Dispersion slope curves as a function of wavelength of the DC-PCFs with $d_{1} / \Lambda=0.3, d / \Lambda=0.8$ and different $\Lambda$ values.

Fig. 6 corresponds to the residual dispersion obtained after the dispersion compensation by $3.9 \mathrm{~km}$ long PCF for the dispersion accumulated in one span (80 $\mathrm{km}$ ) of SMF. It can be observed that the residual dispersion is $\pm 110 \mathrm{ps} / \mathrm{nm}$, which is within the range of $\pm 1000 \mathrm{ps} / \mathrm{nm}$, enabling the proposed PCF structure to be suitable for $10 \mathrm{~Gb} / \mathrm{s}$ transmission system [13].

Fig. 7 represents that in the spectral range $1.46-1.64 \mu \mathrm{m}$ wavelength, the values of dispersion slope are between -1.15 and $-1.45 \mathrm{ps} /\left(\mathrm{nm}^{2} . \mathrm{km}\right)$ for the optimum design parameters: $d_{1} / \Lambda=0.3, d / \Lambda=0.8$ and $\Lambda=0.78 \mu \mathrm{m}$.

Fig. 8 depicts the proposed DC-PCFs RDS value $0.0036 \mathrm{~nm}^{-1}$ at $1.55 \mu \mathrm{m}$ wavelength which perfectly match to SMFs of $0.0036 \mathrm{~nm}^{-1}$. 


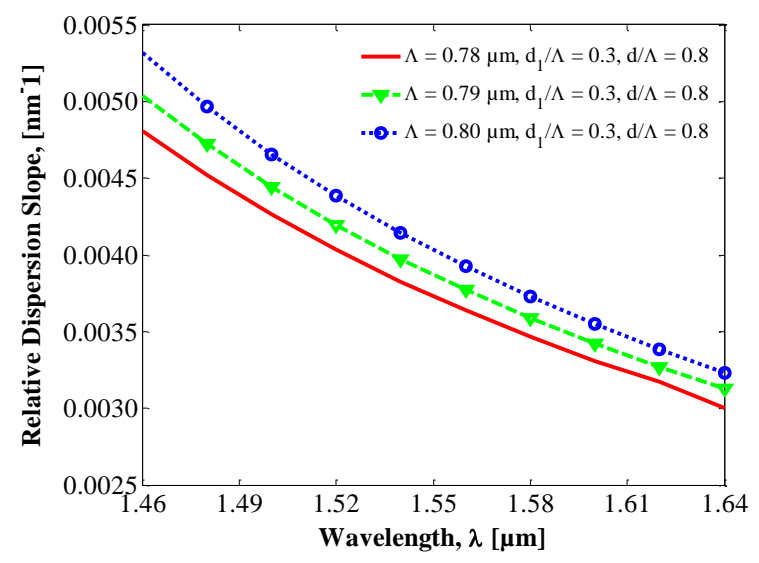

Fig. 8. Relative dispersion slope curves as a function of wavelength of the DCPCFs with $d_{1} / \Lambda=0.3, d / \Lambda=0.8$ and different $\Lambda$ values.

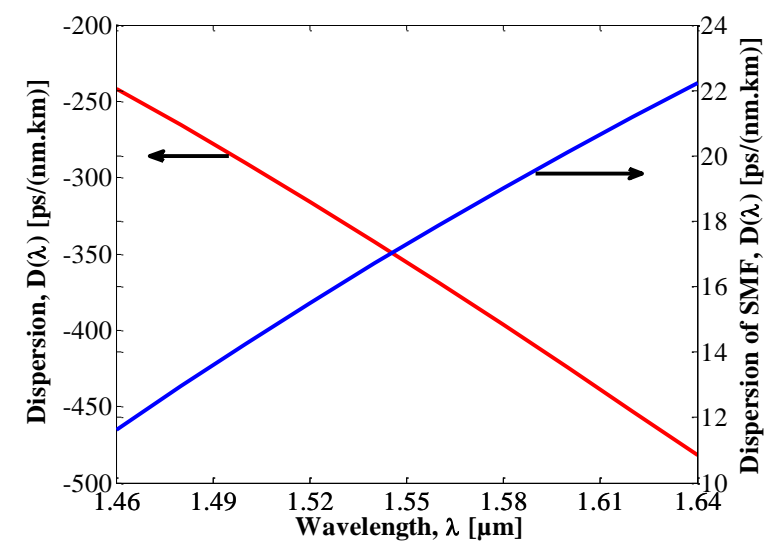

Fig. 9. Dispersion curve for the optimum design parameters: $d_{1} / \Lambda=0.3, d / \Lambda=0.8$ and $\Lambda=0.78 \mu \mathrm{m}$ and dispersion of SMF.

Fig. 9 shows the dispersion curve for the optimum design parameters: $d_{1} / \Lambda=0.3$, $d / \Lambda=0.8$ and $\Lambda=0.78 \mu \mathrm{m}$ and dispersion of SMF. It is revealed from Fig. 8 that the dispersion of SMF is $17 \mathrm{ps} /(\mathrm{nm} . \mathrm{km})$ whereas dispersion of the proposed fiber is $-360 \mathrm{ps} /(\mathrm{nm} . \mathrm{km})$ at $1.55 \mu \mathrm{m}$. So the length of the DCF can be only $4.7 \%$ of the length of SMF to completely compensate the anomalous dispersion at $1.55 \mu \mathrm{m}$. It can be clearly seen that for the optimized set of parameters and air hole ring configurations, a large negative dispersion can be obtained which monotonically decreases over the telecommunication bands and possesses negative dispersion slope, providing good dispersion compensation. 


\section{Conclusion}

In this paper, we have investigated the negative chromatic dispersion coefficient of novel DC-PCFs for broadband dispersion compensation by using FEM. It was found that by adjusting structural parameters of the fiber, broadband DC-PCFs with large negative dispersion over $180 \mathrm{~nm}$ range, better dispersion slope, and better compensation ratio is obtained. Another main advantage is that compared with previously presented DC- PCFs, the design procedure for this proposed DCPCFs structure could be more efficient, easier because relatively lesser geometrical parameters and simple cladding design are needed to be optimized. The proposed novel DC-PCFs can be potential candidate for dispersion compensation in $10 \mathrm{~Gb} / \mathrm{s}$ DWDM optical communication systems.

\section{References}

[1] T.A.Birks,J.C.Knight, B.J. Mangan, P.St.J. Russell, "Photonic crystal fibers: An endless variety", IEICE Trans. Electron. E84-C, 2001, 585-592.

[2] F. Begum, Y. Namihira, S.M.A. Razzak, N. Zou. Novel, "Square Photonic Crystal Fibers with Ultra-Flattened Chromatic Dispersion and Low Confinement Losses”, IEICE Trans. Electron. E90-C, 2007, 607-612.

[3] L.P. Shen, W.P. Huang, S.S. Jian, "Design and optimization of photonic crystal fibers for broadband dispersion compensation", IEEE Photon. Technol. Lett. 15, 2003, 540-542.

[4] J. Laegsgaard, S.E. BarkouLibori, K. Hougaard, J. Riished, T.T. Larsen, T. Sorensen, T.P. Hansen, K.P. Hansen, M.D. Nielsen, J.B. Jensen, and A. Bjarklev, "Dispersion properties of Photonic crystal fibers-Issues and Opportunities”, Materials Research Society Symp. Proc. 797, 2004, W7.1.1W7.1.12.

[5] L. Gruner-Nielsen, S.N. Knudsen, B. Edvold, T. Veng, D. Magnussen, C.C.Larsen, and H. Damsgaard, "Dispersion compensating fibers", Opt. Fiber Technol. 6, , 2000, 164-180.

[6] Y. Ni, L. Zhang, L. An, J. Peng, C.C. Fan, "Dual-core photonic crystal fiber for dispersion compensation" Photon. Tech. Lett. 16, 2004, 1516-1518.

[7] F. Gérôme, J.L. Auguste, J.M. Blondy, "Design of dispersion-compensating fibers based on a dual-concentric-core photonic crystal fiber" Opt. Lett. 29, 2004, 2725-21727.

[8] S.G.Yang, Y.J. Zhang, L.N. He, S.Z. Xie, "Broadband dispersioncompensating photonic crystal fiber. Opt. Lett. 31, 2006, 2830-2832.

[9] A. Huttunen, P. Torma, "Optimization of dual-core and microstructure fiber geometries for dispersion compensation and large mode area”. Opt. Exp. 13, 2005, 627-635. 
[10] F. Gérôme, J.L. Auguste, S. Février, J. Maury, J.M. Blondy, L. Gasca, and L. Provost, "Dual concentric core dispersion compensating fiber optimized for WDM application", Elec. Lett. 41, 2005, 116-117.

[11] Nuyts RJ, Park YK, Gallion P., "Dispersion equalization of a $10 \mathrm{~Gb} / \mathrm{s}$ repeater transmission system using dispersion compensating fibers", J Lightwave Technol : 31,1997.

[12] K. Saitoh and M. Koshiba, "Full-vectorial imaginary-distance beam propagation method based on a finite element scheme: Application to photonic crystal fibers", IEEE J. Quantum Electron., vol. 38, no. 7, 2002, pp. 927-933.

[13] M. N. Islam, 2004. Raman Amplification for Telecommunications 1 and 2 (Springer-Verlag, New York).

[14] T. Matsui, J. Zhou, K. Nakajima and I. Sankawa, "Dispersion flattened photonic crystal fiber with large effective area and low confinement loss", J. Lightwave Technol. 23(12): 2005, 4178-4183. 